\title{
ÜBER DIE EXTREMALEN POLYNOME EINES DURCH ZWEI KNOTEN UNTERTEILTEN KREISES
}

\section{B. GERMANSKY}

1. Die Polynome von Jacobi. Wir wollen hier in einem bescheidenen Masse die Theorie der Polynome entwickeln, die im wesentlichen in demselben Verhältnis zum Einheitskreis $C:|z|=1$ stehen, wie die Jacobischen Polynome zur Einheitsstrecke $I:[-1,+1]$.

Wir schicken einiges über die klassischen Jacobischen Polynome voraus, die von uns in einem anderen Aufsatz [1, p. 953] behandelt worden sind. Dieselben können wie bekannt $[2$, p. 139, Theorem 6.7.1] als diejenigen Polynome

$$
P_{n}^{(\alpha, \beta)}(x)=\left(x-x_{1}^{(n)}\right)\left(x-x_{2}^{(n)}\right) \cdots\left(x-x_{n}^{(n)}\right)
$$

definiert werden, deren Nullstellen $x_{1}^{(n)}, x_{2}^{(n)}, \cdots, x_{n}^{(n)}$ diejenigen Werte der Variablen $x_{1}, x_{2}, \cdots, x_{n}$ sind, die die Funktion

$$
U_{n}\left(x_{1}, x_{2}, \cdots, x_{n}\right)=\prod_{k=1}^{n}\left(1-x_{k}\right)^{p}\left(1+x_{k}\right)^{q} \prod_{1 \leq v<\mu \leq n}\left(x_{\mu}-x_{v}\right)
$$

auf dem Intervall $I$ maximieren. (Die Eindeutigkeit ist bis auf einen belanglosen konstanten Faktor gesichert [2, p. 139].) Hier sind die Gewichte (oder elektrischen Ladungen) $p$ und $q$ mit den Parametern $\alpha$ und $\beta$ durch die Beziehungen $\alpha=2 p-1$ und $\beta=2 q-1$ verknüpft und $x_{1}>x_{2}>\cdots>x_{n}$ vorausgesetzt.

Nun ist aber die Funktion (2) nur ein Faktor der Funktion

$$
V_{m}\left(\xi_{1}, \xi_{2}, \cdots, \xi_{m} ; e_{1}, e_{2}, \cdots, e_{m}\right)=\prod_{1 \leq i<j \leq m}\left(\xi_{j}-\xi_{i}\right)^{e_{i e} e_{j}},
$$

wo $\xi_{1}=-1, \xi_{2}=x_{1}, \xi_{3}=x_{2}, \cdots, \xi_{m-1}=x_{n}, \xi_{m}=+1 ; \xi_{1}<\xi_{2}<\cdots<\xi_{m}$; $e_{1}=q, e_{2}=e_{3}=\cdots=e_{m-1}=1, e_{m}=p, m=n+2$, nämlich derjenige Faktor, der nur die in bezug auf die Maximierung von (3) variablen Argumente $\xi_{2}, \xi_{3}, \cdots, \xi_{m-1}$ der Funktion (3) enthält, während der übrigbleibende konstante Faktor $2^{p q}$ fortgelassen ist. ${ }^{1}$ Daher ist die Maximierung der Funktion (3), die wir die verallgemeinerte Vandermonde nennen, in demjenigen Sinne der Maximierung der Funktion (2) äquivalent, dass das Punktsystem $\xi_{2}^{(m)}=x_{1}^{(n)}, \quad \xi_{3}^{(m)}=x_{2}^{(n)}, \cdots$,

Received by the editors January 23, 1961 and, in revised form, April 10, 1961 and May 11, 1961.

${ }^{1}$ Wir sind zwangsläufig dazu gebracht worden, die Variablen einerseits durch $x_{1}, x_{2}, \cdots, x_{n}$ und andererseits durch $\xi_{2}, \xi_{3}, \cdots, \xi_{m-1}$ zu bezeichnen, da wir die Funktion (3) anstatt (2) benutzen wollen, und deren Argumente auch Konstanten enthalten. 
$\xi_{m-1}^{(m)}=x_{n}^{(n)}$, das die erstere Funktion maximiert, dasselbe ist, das die letztere maximiert. Da überdies die Funktion (3) augenscheinlich einfacher und symmetrischer gebaut und deshalb übersichtlicher als (2) ist, ziehen wir vor, mit der Funktion (3) als unserer Grundfunktion zur arbeiten. Dementsprechend sind die Jacobischen Polynome (1), die wir jetzt in der Form $P_{n}^{\left(\alpha_{1}, \alpha_{2}\right)}(x)$, indem wir $\alpha=\alpha_{2}$ und $\beta=\alpha_{1}$ setzen, schreiben, jetzt als diejenigen Polynome definiert, deren Nullstellen $\xi_{2}^{(m)}=x_{1}^{(n)}, \xi_{3}^{(m)}=x_{2}^{(n)}, \cdots, \xi_{m-1}^{(m)}=x_{n}^{(n)}$ diejenigen Werte der Variablen $\xi_{2}=x_{1}, \xi_{3}=x_{2}, \cdots, \xi_{m-1}=x_{n}$ sind, die die Funktion (3) auf $I$ maximieren. ${ }^{2}$ Wir schreiben auch $e_{1}=q=\rho_{1}$ und $e_{m}=p=\rho_{2}$ und haben $\alpha_{1}=2 \rho_{1}-1$ und $\alpha_{2}=2 \rho_{2}-1$. Die Argumente $\xi_{1}=-1$ und $\xi_{m}=+1$, die wir auch mit $a_{1}$ bzw. $a_{2}$ bezeichnen, nennen wir die Fixstellen der Funktion (3). Wir sagen, dass (3) vom Range (der gesamten Anzahl ihrer Argumente $\left.\xi_{1}, \xi_{2}, \cdots, \xi_{m}\right) m=n+2$ ist.

Wir nennen das System $(\xi ; e)$ einer Koordinate $\xi$ und des zu ihr gehörigen Gewichtes $e$ einen physikalischen Punkt $P(\xi ; e)$ und wenn dieser Punkt fest ist, einen Knoten. Auf diese Weise sind die klassischen Jacobischen Polynome dadurch charakterisiert dass die zu ihnen gehörige Grundmenge $I$ durch die beiden Knoten $A_{1}\left(a_{1} ; \rho_{1}\right)$ und $A_{2}\left(a_{2} ; \rho_{2}\right)$ berandet ist.

2. Die extremalen Polynome eines von zwei Knoten berandeten Kreisbogens. Wenn wir die obigen Betrachtungen auf den Fall eines Kreisbogens ausdehnen wollen, dann müssen wir die $n$ Argumente $\zeta_{2}=z_{1}, \zeta_{3}=z_{2}, \cdots, \zeta_{m-1}=z_{n}$ des absoluten Betrages

$$
\left|V_{m}\left(\zeta_{1}, \zeta_{2}, \cdots, \zeta_{m} ; e_{1}, e_{2}, \cdots, e_{m}\right)\right|=\prod_{1 \leq i<j \leq m}\left|\zeta_{j}-\zeta_{i}\right| e_{i e j}
$$

der verallgemeinerten Vandermonde $V_{m}\left(\zeta_{1}, \zeta_{2}, \cdots, \zeta_{m} ; e_{1}, e_{2}, \cdots, e_{m}\right)$ $=\prod_{1 \leq i<j \leq m}\left(\zeta_{j}-\zeta_{i}\right)^{e_{i} e_{j}}$ des Ranges $m$ (die bis auf die Bezeichnung der Argumente dieselbe wie die Funktion (3) ist), zwecks dessen Maximierung auf einem Einheitskreisbogen, der durch die beiden Knoten $A_{1}\left(a_{1} ; \rho_{1}\right)$ und $A_{2}\left(a_{2} ; \rho_{2}\right)$ berandet ist, varieren lassen. Wir benutzen jetzt die Buchstaben $\zeta$ und $z$ anstatt $\xi$ bzw. $x$ zur Andeutung des Umstandes, dass wir es jetzt mit komplexen an Stelle von reellen Argumenten der verallgemeinerten Vandermonde zu tun haben. Wie im Falle der Jacobischen Polynome haben wir den $\boldsymbol{n}$ variablen Punk-

2 Wir haben in dem obigen Aufsatz $1 \alpha_{0}$ für $\alpha_{1}$ und $\alpha_{1}$ für $\alpha_{2}$ geschrieben. Doch scheint es besser zu sein, so zu schreiben, wie jetzt. Dadurch bekommen die gewöhnlichen Jacobischen Polynome die Ordnung 2, entsprechend der Anzahl zwei ihrer Fixstellen. Die Ordnung eines allgemeinen Extremalpolynoms wird überhaupt als die Anzahl der zu ihm gehörigen Fixstellen oder Knoten der entsprechenden verallgemeinerten Vandermonde, definiert Daher haben die in diesem Aufsatz betrachteten Extremalpolynome des durch zwei Knoten unterteilten Kreises die Ordnung 2. 
ten $\zeta_{2}=z_{1}, \zeta_{3}=z_{2}, \cdots, \zeta_{m-1}=z_{n}$ das Gewicht (die Ladung) 1 und den beiden festen Punkten $\zeta_{1}=a_{1}$ und $\zeta_{m}=a_{2}$ die willkürlichen Gewichte $e_{1}=\rho_{1}>0$ und $e_{m}=\rho_{2}>0$ zuzuschreiben. Hierdurch stellt sich aber heraus, dass das neue Problem nicht dem klassischen Jacobischen Problem völlig analog ist, wie im nächsten Paragraphen gezeigt wird.

Wir schreiben die neuen Polynome, die dadurch charakterisiert sind, dass ihre Nullstellen $z_{1}^{(n)}=\zeta_{2}^{(m)}, z_{2}^{(n)}=\zeta_{3}^{(m)}, \cdots, z_{n}^{(n)}=\zeta_{m-1}^{(m)}$ diejenigen Werte der $n$ Argumente $z_{1}=\zeta_{2}=e^{i \phi_{1}}, z_{2}=\zeta_{3}=e^{i \phi_{2}}, \cdots, z_{n}$ $=\zeta_{m-1}=e^{i \phi_{n}}$ der verallgemeinerten Vandermonde (4) sind, die deren absoluten Betrag auf dem in Frage stehenden Kreisbogen unter der Bedingung maximieren, dass ihre beiden anderen Argumente $\zeta_{1}=a_{1}$ $=e^{i \theta_{1}}$ und $\zeta_{m}=a_{2}=e^{i \theta_{2}}$ fest bleiben, in der Form

$$
Q_{n}^{\left(\alpha_{1}, \alpha_{2}\right)}(z)=\left(z-z_{1}^{(n)}\right)\left(z-z_{2}^{(n)}\right) \cdots\left(z-z_{n}^{(n)}\right) .
$$

Wieder gilt $\alpha_{1}=2 \rho_{1}-1$ und $\alpha_{2}=2 \rho_{2}-1$.

3. Die konjugierten extremalen Polynome des durch zwei Knoten unterteilten Einheitskreises. Es stellt sich heraus, dass unser neues Problem sich nicht ändert, wenn wir anstatt alle $n$ Punkte $P_{1}\left(z_{1}\right)$, $P_{2}\left(z_{2}\right), \cdots, P_{n}\left(z_{n}\right)$ auf dem durch die beiden Knoten $A_{1}\left(a_{1} ; \rho_{1}\right)$ und $A_{2}\left(a_{2} ; \rho_{2}\right)$ berandeten Bogen variieren zu lassen, dieselben irgendwie auf die beiden komplementären Kreisbögen $a_{1} a_{2}$ und $a_{2} a_{1}$ des Einheitskreises verteilen und dort variieren lassen. In der Tat sind ja die beiden Bögen analytisch, in bezug auf ihre Gleichung, nicht voneinander $z u$ unterscheiden und die Punkte $z_{1}^{(n)}, z_{2}^{(n)}, \cdots, z_{n}^{(n)}$ bleiben auch in den neuen Fällen innere Punkte der betreffenden Kreisbögen. Demnach bleiben die Bedingungen für das Eintreten eines Maximums des absoluten Betrages der verallgemeinerten Vandermonde dieselben wie im Falle der Verteilung der $\boldsymbol{n}$ variablen Punkte bloss auf dem Bogen $a_{1} a_{2}$. Dadurch aber erscheinen als Lösungen unseres Problems insgesamt mindestens $n+1$ Punksysteme $z_{1}^{(n)}, z_{2}^{(n)}, \cdots, z_{n}^{(n)}$, nämlich mindestens eines, dessen Glieder alle dem Kreisbogen $a_{1} a_{2}$, mindestens eines, dessen Glieder $z_{1}^{(n)}, z_{2}^{(n)}, \cdots, z_{n-1}^{(n)}$ dem Kreisbogen $a_{1} a_{2}$ und das Glied $z_{n}^{(n)}$ dem Kreisbogen $a_{2} a_{1}$ usf. bis zu dem Falle, dass dessen alle Glieder dem komplementären Kreisbogen $a_{2} a_{1}$ angehören. Denn zu jeder Verteilung der Punkte $z_{1}, z_{2}, \cdots, z_{n}$ auf die beiden komplementären Kreisbögen $a_{1} a_{2}$ und $a_{2} a_{1}$ (der Kreis ist als orientiert, z.B. entgegengesetzt dem Uhrzeigersinne, anzunehmen) gehört, wie leicht ersichtlich, mindestens ein besonderes Maximum des absoluten Betrages der verallgemeinerten Vandermonde auf dem Kreise. Alle diese Punktsysteme und die zugehörigen Polynome $\left(z-z_{1}^{(n)}\right)\left(z-z_{2}^{(n)}\right)$ ... $\left(z-z_{n}^{(n)}\right)$ sind gleichberechtigt und wir behalten für die letzteren 
die Bezeichnung (5). Wir leiten im nächsten Paragraphen auch eine gemeinsame Differentialgleichung (mit diskreten Eigenwerten, die der Multiplizität $\geqq n+1$ der Polynome Rechnung tragen, versteht sich) für alle diese Polynome.

Es ist bewiesen [3], dass im Falle $\rho_{1}=\rho_{2}=1 \mathrm{zu}$ jeder Verteilung $\left(n_{1}, n_{2}\right), n_{1}+n_{2}=n$, der $n$ Punkte $z_{1}, z_{2}, \cdots, z_{n}$ auf die beiden Bögen des Kreises $|z|=1$ ( $n_{1}$ auf den Bogen $a_{1} a_{2}$ und $n_{2}$ auf den Komplementärbogen $a_{2} a_{1}$ ) nur ein einziges Punktsystem $z_{1}^{(n)}, z_{2}^{(n)}, \cdots, z_{n}^{(n)}$ existiert, das die Funktion (4) im obigen Sinne, d.h. unter Festhaltung der Punkte $A_{1}\left(a_{1} ; \rho_{1}\right)$ und $A_{2}\left(a_{2} ; \rho_{2}\right)$, maximiert.

Wir nennen die (mindestens $n+1)$ Punksysteme $z_{1}^{(n)}, z_{2}^{(n)}, \cdots, z_{n}^{(n)}$ und Polynome $Q_{n}^{\left(\alpha_{1}, \alpha_{2}\right)}(z)$ die extremalen Punktsysteme und Polynome des durch zwei Knoten unterteilten Einheitskreises. Die mindestens $n+1$ Polynome $Q_{n}^{\left(\alpha_{1}, \alpha_{2}\right)}(z)$ des festen Grades $n$ nennen wir zueinander konjugiert oder assoziiert. Die Verteilung $\left(n_{1}, n_{2}\right)$ heisst nach Szegö [2, p. 152] der Typus sowohl der Punktsysteme $z_{1}^{(n)}, z_{2}^{(n)}$, $\cdots, z_{n}^{(n)}$ und $\zeta_{1}^{(m)}, \zeta_{2}^{(m)}, \cdots, \zeta_{m}^{(m)}$ als auch des Polynoms $Q_{n}^{\left(\alpha_{1}, \alpha_{2}\right)}(z)$ in Frage. Das Punktsystem $\zeta_{1}^{(m)}, \zeta_{2}^{(m)}, \cdots, \zeta_{m}^{(m)}$ heisst ein erweitertes extremales Punktsystem und das Punktsystem $z_{1}^{(n)}, z_{2}^{(n)}, \cdots, z_{n}^{(n)}$ heisst ein eingeschränktes extremales Punktsystem, zum unterteilten Kreis gehörig. Ähnlich wird das Polynom $Q_{n}^{\left(\alpha_{1}, \alpha_{2}\right)}(z)$ ein eingeschränktes extremales Polynom und das "Polynom"

$$
q_{m}^{\left(\alpha_{1}, \alpha_{2}\right)}(z)=\left(z-a_{1}\right)^{\rho_{1}} Q_{n}^{\left(\alpha_{1}, \alpha_{2}\right)}(z)\left(z-a_{2}\right)^{\rho_{2}}
$$

ein erweitertes extremales "Polynom," zum unterteilten Kreis gehörig genannt.

4. Dir Differentialgleichung der konjugierten extremalen Polynome des durch zwei Knoten unterteilten Kreises. Durch logarithmische Differentiation des Ausdruckes

$$
\begin{aligned}
\left|V_{m}\left(\zeta_{1}, \zeta_{2}, \cdots, \zeta_{m} ; e_{1}, e_{2}, \cdots, e_{m}\right)\right|^{2} & \\
= & \left|V_{m}\left(e^{i \phi_{1}}, e^{i \phi_{2}}, \cdots, e^{i \phi_{n}}, a_{1}, a_{2} ; \rho_{1}, \rho_{2}\right)\right|^{2}
\end{aligned}
$$

nach den Variablen $\phi_{1}, \phi_{2}, \cdots, \phi_{n}$, erhalten wir nach einiger Rechnung

(7) $\sum_{\nu=1}^{n} \frac{2}{z_{k}^{(n)}-z_{v}^{(n)}}+\frac{2 \rho_{1}}{z_{k}^{(n)}-a_{1}}+\frac{2 \rho_{2}}{z_{k}^{(n)}-a_{2}}-\frac{\rho_{1}+\rho_{2}+n-1}{z_{k}^{(n)}}=0$,

$$
k=1,2, \cdots, n \text {. }
$$

Dabei haben wir auf der rechten Seite der Gleichung (6) nur diejenigen der Ladungsargumente explizit angeführt, die wirklich verän- 
derlich (nicht gleich 1) sind, nämlich $\rho_{1}$ und $\rho_{2}$. Ausserdem braucht die geschriebene Ordnung der Punktargumente $e^{i \phi_{1}}, \cdots, e^{i \phi_{n}}, a_{1}, a_{2}$ auf der rechten Seite der Gleichung (6) nicht dieselbe zu sein, wie auf deren linker Seite und wie die tatsächliche Ordnung der Punkte auf dem Einheitskreis. Die Funktion (6) ist ja sowieso symmetrisch in den Argumenten $A_{1}\left(a_{1} ; \rho_{1}\right), P_{1}\left(z_{1}\right), \cdots, P_{n}\left(z_{n}\right), A_{2}\left(a_{2} ; \rho_{2}\right)$. Durch Einführung von $Q_{n}(z)=\left(z-z_{1}^{(n)}\right)\left(z-z_{2}^{(n)}\right) \cdots\left(z-z_{n}^{(n)}\right)$ in $(7)$ erhalten wir

$$
\frac{Q_{n}^{\prime \prime}\left(z_{k}^{(n)}\right)}{Q_{n}^{\prime}\left(z_{k}^{(n)}\right)}+\frac{2 \rho_{1}}{z_{k}^{(n)}-a_{1}}+\frac{2 \rho_{2}}{z_{k}^{(n)}-a_{2}}-\frac{\rho_{1}+\rho_{2}+n-1}{z_{k}^{(n)}}=0
$$

oder

$$
\begin{aligned}
& \left(z_{k}^{(n)}-a_{1}\right)\left(z_{k}^{(n)}-a_{2}\right) z_{k}^{(n)} Q_{n}^{\prime \prime}\left(t_{k}\right) \\
& \quad+\left[2 \rho_{1}\left(z_{k}^{(n)}-a_{2}\right) z_{k}^{(n)}+2 \rho_{2}\left(z_{k}^{(n)}-a_{1}\right) z_{k}^{(n)}\right. \\
& \left.\quad-\left(\rho_{1}+\rho_{2}+n-1\right)\left(z_{k}^{(n)}+a_{1}\right)\left(z_{k}^{(n)}-a_{2}\right)\right] Q_{n}^{\prime}\left(z_{k}^{(n)}\right)=0 .
\end{aligned}
$$

Wir haben das Resultat erhalten, dass das Polynom

$$
\begin{aligned}
\left(z-a_{1}\right)\left(z-a_{2}\right) z Q_{n}^{\prime \prime}(z) & +\left[2 \rho_{1}\left(z-a_{2}\right) z+2 \rho_{2}\left(z-a_{1}\right) z\right. \\
- & \left.\left(\rho_{1}+\rho_{2}+n-1\right)\left(z-a_{1}\right)\left(z-a_{2}\right)\right] Q_{n}^{\prime}(z)
\end{aligned}
$$

vom Grade $n+1$ die $n$ Nullstellen $z_{1}^{(n)}, z_{2}^{(n)}, \cdots, z_{n}^{(n)}$ gemeinsam mit dem Polynom (5) hat, und somit durch dasselbe teilbar ist. Daher ist ähnlich wie bei Szegö [2, p. 152, paragraph 6.82]

$$
\begin{aligned}
& \left(z-a_{1}\right)\left(z-a_{2}\right) z u^{\prime \prime}+2\left[\rho_{1}\left(z-a_{2}\right) z+\rho_{2}\left(z-a_{1}\right) z\right. \\
& \left.-\frac{\rho_{1}+\rho_{2}+n-1}{2}\left(z-a_{1}\right)\left(z-a_{2}\right)\right] u^{\prime}-(\gamma z+\delta) u=0
\end{aligned}
$$

wo $\gamma z+\delta$ ein geeignetes Polynom ersten Grades (der Quotient der beiden Polynome (10) und (5)) ist, das so zu bestimmen ist, dass (11) eine Polynomlösung vom Grade $n$ hat. Diese Lösung kann auch so charakterisiert werden, dass sie in der ganzen Ebene stetig ist, und dass sie im Randpunkt $\infty$ die Beziehung $z^{-n} Q_{n}(z)=O_{z \rightarrow \infty}(1)$ erfüllt, wobei $O(1)$ das bekannte Landausche Symbol ist. Daher sind $\gamma$ und $\delta$ Eigenwertparameter, deren Anzahl in diesem Falle 2 beträgt. Das Polynom $\gamma z+\delta$ ist vom Herrn Professor E. Mohr ein Eigenwertpolynom genannt worden.

(11) stellt die Differentialgleichung für alle eingeschränkten Ex- 
tremalpolynome vom festen Grad $n$ des durch die zwei Knoten $\left(a_{1} ; \rho_{1}\right)$ und $\left(a_{2} ; \rho_{2}\right)$ unterteilten Einheitskreises dar. Da der Typus der Lösungspolynome in dieser Differentialgleichung nicht vorkommt, ist dieselbe allen konjugierten Polynomen desselben Grades gemeinsam. Natürlich lässt die Differentialgleichung (11) auch andere Formen zu. So ist es z.B. möglich eine Differentialgleichung für die erweiterten "Extremalpolynome" aus (11) abzuleiten. Doch gehen wir darauf nicht mehr ein.

Die Differentialgleichung (11) ist von der allgemeinen Form

$$
A(z) u^{\prime \prime}+2 B_{n}(z) u^{\prime}+C_{n}(z) u=0
$$

wo

$$
A(z)=\left(z-a_{1}\right)\left(z-a_{2}\right) z
$$

und

$$
\frac{B_{n}(z)}{A(z)}=\frac{\rho_{1}}{z-a_{1}}+\frac{\rho_{2}}{z-a_{2}}-\frac{\rho_{1}+\rho_{2}+n-1}{2 z}
$$

ist.

Wählen wir die beiden Fixstellen der verallgemeinerten Vandermonde, $a_{1}$ und $a_{2}$, konjugiert komplex zueinander; $a_{2}=\bar{a}_{1}$, was ohne Beeinträchtigung der Allgemeinheit unserer Ausführungen möglich ist, dan werden alle Polynome $Q_{n}^{\left(\alpha_{1}, \alpha_{2}\right)}(z)$ reelle Polynome.

5. Schlussbemerkungen. Die Differentialgleichung (11) ist insofern nicht vom üblichen Typus, als der Koeffizient von $u^{\prime}$ den Grad $\boldsymbol{n}$ ihrer Lösungspolynome enthält. Dies macht ihre Behandlung viel schwieriger als die Behandlung ähnlicher Differentialgleichungen, die diese Eigenschaft nicht haben, z.B. der Differentialgleichung für die verallgemeinerten Jacobischen Polynome $[1$, p. 955]. Durch denselben Umstand, sowie durch die Tatsache, dass ihre singulären Punkte wenigstens zum Teil komplex sind, unterscheidet sie sich auch von der Heunschen Differentialgleichung [4].

Aus den genannten Gründen ist nicht zu erwarten, dass die Lösungspolynome der Differentialgleichung (11) zueinander im üblichen Sinne orthogonal sind. Dies mag die Ursache sein, weshalb unser Problem bis heute nicht behandelt worden ist. Die Frage, was die verallgemeinerte Orthogonalitätsrelation $[1$, p. 956] der extremalen Polynome des durch zwei Knoten (und noch mehr des durch mehr als zwei Knoten) unterteilten Kreises ist, muss von uns offen gelassen werden. Doch scheint deren Existenz durch den eben zitierten Aufsatz gesichert zu sein. 


\section{BIBLIOGRAPHY}

1. B. Germansky, An identity in the theory of the generalized polynomials of Jacobi, Proc. Amer. Math. Soc. 9 (1958), 953-956.

2. G. Szegö, Orthogonal polynomials, New York, 1959.

3. B. Germansky, On the Fekete systems of an arc of a circle, Riveon Lematematika 3 (1949), 6 (in hebraisch, mit einer Zusammenfassung in englisch).

4. Karl Heun, $Z$ ur Theorie der Riemannschen Funktionen zweiter Ordnung mit vier Verzweigungspunkten, Math. Ann. 33 (1889), 165, Gleichung (10).

Mathematical Institute,

TeChNical UNiversity OF BerLin

\section{ON REDUNDANCY OF POWERS AND THE MOMENT PROBLEM}

DAVID S. GREENSTEIN

1. Introduction. Let $\psi(t)$ be a bounded nondecreasing function with infinitely many points of increase such that the mass distribution $d \psi(t)$ has moments of all orders. Under these conditions, the powers $t^{n}(n=0,1, \cdots)$ are linearly independent elements of $L^{2} \psi$, which consists of all $\psi$-measurable $f(t)$ for which

$$
\|f(t)\|_{\psi}^{2}=\int_{-\infty}^{\infty}|f(t)|^{2} d \psi(t)<\infty .
$$

We shall call any of the above powers redundant if it belongs to the closed linear manifold of $L_{\psi}^{2}$ spanned by all higher powers. The author has shown [2, Theorem 2.4] that, for absolutely continuous $d \psi(t)$, if the powers span $L^{2} \psi$, then each power is redundant. This result, while weaker than the Müntz-Szasz Theorem, does not require that $d \psi(t)$ have compact support.

In the present paper, redundancy is studied for general $d \psi(t)$, thus placing the above result in a more natural context. Our investigation, using the theory of the Hamburger moment problem, allows us to study some properties of extremal solutions of the moment problem and to add to Wright's work [6] on backward extension of moment sequences.

2. Some needed results. The following theorem of M. Riesz $\left[4 ; 5\right.$, p. 60] is important for polynomial approximation in $L_{\psi}^{2}$ :

Received by the editors March 6, 1961. 